\title{
Die Schließung des Instituts für Molekularbiologie in Salzburg - Versuch einer Chronik
}

\author{
Von \\ Günther Kreil \\ (Vorgelegt in der Sitzung der math.-nat. Klasse am 15. Dezember 2005 \\ durch das w. M. Günther Kreil)
}

\section{Einleitung}

Vor rund zwei Jahren wurde das Institut für Molekularbiologie in Salzburg, immerhin das zweitgrößte Akademie-Institut, geschlossen. Aus Gesprächen mit Akademie-Mitgliedern und Außenstehenden weiß ich, dass über die Gründe für diesen Schritt gerätselt wird. Die Frage „Wie konnte es dazu kommen?" oder die Feststellung „Das hätte man doch verhindern müssen“" waren häufig Teil solcher Diskussionen. Ich bin der Meinung, dass mehrere Faktoren, interne und externe, letztlich dazu führten, dass es zu dieser Schließung kam. Ein Ereignis dieses Ausmaßes hat es meines Wissens in der Geschichte der Akademie-Institute noch nie gegeben, was allein schon ein ausreichender Grund wäre, die Entwicklungen, die zu diesem Schritt führten und die ich als Zeuge und indirekt auch als Betroffener erlebte, zu beschreiben. In diesem Beitrag möchte ich demnach versuchen, die letzten Jahre des Instituts aus persönlicher Sicht zu dokumentieren. Von einigen früheren Mitarbeitern erhielt ich detaillierte Unterlagen zu einzelnen Aspekten, die ich aber schon aus Platzgründen nur zu einem kleinen Teil verwendet habe. 


\section{Kurze Geschichte des Instituts}

Bei der Initiative zur Gründung des Instituts für Molekularbiologie haben die w. M. Kratky, Tuppy und Wessely, unterstützt durch den aus Österreich stammenden Nobelpreisträger Max Perutz, eine wichtige Rolle gespielt. Im April 1966 haben dann zwei Abteilungen ihre Arbeit begonnen, eine in Wien und eine in Graz in Räumen von Universitätsinstituten. Es war ein bescheidener Anfang mit je 1-3 Mitarbeitern. In den folgenden Jahren, in denen diese Abteilungen langsam gewachsen sind, wurde seitens der ÖAW versucht, ein geeignetes Areal für einen Institutsneubau in Wien zu finden. Dies scheiterte, so dass ein Angebot des Grafen Johannes Moy, der ÖAW ein Grundstück in Salzburg zu schenken, dankbar angenommen wurde. Dort war inzwischen ein dritte Abteilung gegründet worden. Im Herbst 1976 konnten die drei Abteilungen Biologie (später Entwicklungsbiologie, Kratochwil), Chemie (Biochemie, Kreil) und Physik (Zellbiologie, Small) das neu erbaute Institutsgebäude beziehen. Im November 1977 wurde des Institut feierlich eröffnet, u. a. mit einem Symposium, an dem rund ein Dutzend Wissenschaftler aus Europa und den USA ihre Arbeiten präsentierten. In der Folgezeit wurden dann zusätzliche Nachwuchsgruppen gegründet, vor allem die des Ehepaars Matzke, die die ganz junge Disziplin der Anwendung der Gentechnik bei pflanzlichen Organismen in Österreich etablierten. Bei all diesen und den weiteren Entwicklungen spielte w. M. Tuppy als langjähriger Vorsitzender des Kuratoriums eine besonders wichtige Rolle.

Die wissenschaftlichen Arbeiten am Institut fanden international und national steigende Anerkennung, was sich unter anderem darin manifestierte, dass drei Wissenschaftler zu Mitgliedern der EMBO (European Molecular Biology Organisation) gewählt wurden. Hinzu kamen ein wirkliches und zwei korrespondierende Mitglieder der ÖAW, das sind immerhin mehr als in allen anderen AkademieInstituten zusammen. Durch moderat steigende Akademie-Budgets und erfolgreiche Einwerbung von Drittmitteln, auch von Firmen, ist das Institut stetig gewachsen, in den Neunzigerjahren waren es jeweils 50-60 Personen. Zu diesen zählten auch Diplomanden und Dissertanten der Universität Salzburg und anderer österreichischer Universitäten, sowie Postdoktoranden und Gäste aus verschiedenen europäischen und außereuropäischen Ländern. Das Instituts-Budget betrug 2003, im Jahr der Schließung, rund 2,5 Millionen Euro (34 Millionen Schilling). Verglichen mit den im Zusammenhang mit dem neu gegründeten IMBA kolportierten Budgetzahlen war die Mole- 
kularbiologie in Salzburg ein billig und effizient operierendes Institut mit einer, speziell gemessen am Aufwand, hohen wissenschaftlichen Produktivität.

\section{Evaluierungen}

Im Jahre 1995 wurden die biochemischen Wissenschaften in Österreich mit Unterstuitzung des Wissenschaftsministeriums von der EMBO evaluiert. Diese nominierte mehr als ein Dutzend Wissenschaftler aus verschiedenen europäischen Ländern und aus Israel, die nach vielen Interviews, Studium der Unterlagen, Institutsbesuchen usw. letztlich einen fast 300 Seiten umfassenden Bericht vorlegten. Das Institut für Molekularbiologie hat sich selbstverständlich an dieser Evaluierung beteiligt, und das Resultat war insgesamt ein höchst erfreuliches. Die wissenschaftlichen Arbeiten wurden sehr positiv bewertet und deren nach internationalen Maßstäben hoher Standard ausdrücklich gewürdigt.

Im März 1999 fand dann eine weitere, vom Präsidium der ÖAW initiierte Evaluierung des Instituts statt. Vier Gutachter, ein Pflanzenmolekularbiologe aus Tübingen für die Gruppe Matzke und drei Professoren aus Instituten für Pharmakologie und Toxikologie (Würzburg) bzw. Klinische Biochemie (Genf) besuchten das Institut. Wer diese z.T. auf ganz anderen als am Institut bearbeiteten Gebieten tätigen Wissenschaftler ausgewählt hat, wurde uns nicht mitgeteilt.

Die Evaluierung war, mit einigen Einschränkungen bei den Nachwuchsgruppen, wieder sehr positiv. Allerdings wurde im Bericht angemerkt, dass das Institut nicht das Niveau von Max-PlanckInstituten, des IMP oder der Howard-Hughes-Institute in den USA erreiche. Dieser Vergleich ist jedoch nicht fair. Zweifellos wurden auch am Akademie-Institut wissenschaftliche Leistungen erbracht, die sich durchaus im Rahmen dieser Institutionen hätten sehen lassen können. Als langjähriges Mitglied im Wissenschaftlichen Beirat von zwei Max-Planck-Instituten kann ich dies aus persönlicher Erfahrung bestätigen. Diese Institutionen sind jedoch wesentlich größer, ihre finanzielle und personelle Ausstattung entspricht einem Vielfachen dessen, was dem Institut in Salzburg je zur Verfügung stand. Dieses Akademie-Institut war ein sehr angenehmer Ort für wissenschaftliche Forschung, auch mit der Perspektive einer gewissen Langfristigkeit, doch in einem wesentlich bescheidenerem Rahmen.

Die Kritik der Gutachter, das Institut mache einen ,lokal isolierten Eindruck, auch was den Zustrom von Studenten aus der Universität Salzburg betrifft", stimmte nicht. Wir konnten viele der besten 
Studenten der Universität als Diplomanden und Dissertanten gewinnen. Zudem beteiligten sich Wissenschaftler aus dem Institut häufig an diversen Lehrveranstaltungen an der Universität Salzburg. Zu Recht wurde allerdings kritisiert, dass am Institut eine gewisse fachliche Heterogenität herrsche, was bei den zukünftigen Plänen berücksichtigt werden sollte.

Angesichts der bevorstehenden Pensionierung der Abteilungsleiter Kreil und Kratochwil wurden für die Zukunft zwei im Prinzip ähnliche Szenarien diskutiert, je nachdem, ob die Gruppe Matzke nach Wien übersiedelt oder nicht. Interessanterweise ist ein in den folgenden Diskussionen immer wieder verwendetes Argument, das Institut sei zu klein und müsse dringend vergrößert werden, im Evaluierungsbericht nicht erwähnt worden. In einem Abschnitt „Künftige Infrastruktur“ wurde vielmehr ein konkreter Vorschlag zur Neuorganisation im bestehenden Rahmen gemacht, den die Gutachter wie folgt bewerteten: „Eine solche Struktur würde eine ausreichende kritische Masse darstellen“. Das Argument, das Institut müsse unbedingt erweitert werden, wurde meines Wissens erst vom Präsidium bei den Diskussionen mit dem Salzburger Landeshauptmann verwendet.

\section{Interne Probleme}

Die Stimmung am Institut verschlechterte sich allmählich, v. a. im Lauf des Jahres 1999. Ein Grund war der schon lange laufende arbeitsrechtliche Prozess, den die Akademie auf Betreiben von Herrn Small gegen Herrn Sobieszek führte und der letztlich in allen drei Instanzen verloren wurde. Im Haus führte dies zu einer Spaltung in zwei Lager. Die Vorgangsweise der Akademie, vor allem die Entscheidung, den Instanzenweg voll auszuschöpfen, wurde von der Mehrheit der Mitarbeiter abgelehnt. Dies war auch mit ein Grund, dass die Abteilung Zellbiologie, geleitet von Herrn Small, und der Rest des Hauses kaum noch miteinander kommunizierten. Zudem gab es erste Gerüchte, dass das Institut geschlossen werden könnte.

Zum Eklat kam es schließlich bei der Sitzung des Kuratoriums im März 2000. Herr Small forderte damals ultimativ, dass das seit Jahrzehnten praktizierte Rotationsprinzip mit einer jeweils dreijährigen Amtsperiode des Administrativen Direktors beendet werden solle und er unbefristet die Leitung übernehmen möchte. Im Rahmen dieser Rotation - die sich übrigens aus der Sicht der Institutsmitarbeiter mehr als 20 Jahre ausgezeichnet bewährt hatte - wäre ich wieder an der Reihe gewesen, doch ich war zu diesem Zeitpunkt bereits ein Jahr in Pension und nur mehr interimistischer Leiter der 
Abteilung „Biochemie“. Bei der erwähnten Sitzung wurde die vorgesehene nächste Direktorin, Frau Matzke, massiv attackiert, vor allem von der offensichtlich von Herrn Small instruierten Frau Barlow. Diese hat jedoch ihre Arbeit am Institut erst zwei Monate später begonnen, die vorgebrachten, abwertenden Meinungen über Frau Matzke konnten also nicht auf eigener Wahrnehmung beruhen. Es wurde schließlich ein Kompromiss gefunden: Herr Small blieb ein weiteres Jahr Direktor, dann folgte Frau Matzke.

In dieser Sitzung haben die leitenden Angestellten des Instituts wie eine hoffnungslos zerstrittene Gruppe agiert, und bei den Mitgliedern des Kuratoriums konnte zu Recht der Eindruck entstehen, dass in dieser Atmosphäre eine gedeihliche Arbeit erschwert sein würde. Die schon vorhandene Tendenz der Abkapselung der Abteilung Zellbiologie verstärkte sich in der Folgezeit noch weiter. Zudem hat die sich immer konkreter abzeichnende Möglichkeit einer Schließung des Instituts, die ja dann im Oktober 2000 zur Gewissheit wurde, eine Art „Rette sich wer kann“-Mentalität begünstigt. Die Entscheidung, das Institut nicht weiter zu führen, wurde durch die Vorfälle bei dieser Sitzung des Kuratoriums zweifellos erleichtert.

\section{Korrespondenz Präsident Welzig-Landeshauptmann Schausberger}

Im April 2000 besuchte der Salzburger Landeshauptmann Schausberger das Akademie-Institut. Neben kurzen Präsentationen über die wissenschaftliche Arbeit durch die Mitarbeiter wurde der Landeshauptmann auch über die möglichen Pläne der Akademie hinsichtlich Ausbau oder Schließung informiert. Im Laufe dieses Jahres kam es in dieser Sache zu einem Briefwechsel zwischen Präsident Welzig und Landeshauptmann Schausberger. So liest man im Protokoll der Gesamtsitzung vom 16. Juni 2000, Landeshauptmann Schausberger habe mitgeteilt, dass eine etwaige Unterstützung des Ausbaus des Instituts erst nach Vorliegen der von ihm in Auftrag gegebenen Studie „Wissenschafts- und Forschungsleitbild für Salzburg“ geprüft werden könne. In einem Brief vom 29. Juni, von dem eine Kopie am Institut vorhanden war, hat Präsident Welzig dann Überlegungen und Pläne hinsichtlich einer Neugestaltung und der nationalen und internationalen Positionierung des Instituts erörtert. Dabei erwähnte der Präsident auch das Interesse der Gemeinde Wien, dieses „,renommierte Institut“ (sic!) im Wiener Biozentrum anzusiedeln. In einem abschließenden Halbsatz liest man dann: „,... erlaube ich 
mir die Frage, ob das Land Salzburg bereit ist, den Ausbau der Molekularbiologie am Standort Salzburg mit einem einmaligen Betrag von 70 Millionen Schilling zu unterstützen.“

Die distanzierte Haltung des Landes und der Stadt Salzburg gegenüber dem Institut war uns wohl vertraut, die Naturwissenschaften haben in diesem Bundesland traditionell keinen leichten Stand. Eine Förderung in dieser Höhe wäre aber auch in anderen Bundesländern (vielleicht mit Ausnahme Wiens) wohl kaum realisierbar gewesen. Es wurde hier wohl Unmögliches verlangt, um rasch eine Entscheidung herbeizuführen.

In einem Brief des Landeshauptmanns vom 14. September 2000 wurde dann der Vorschlag gemacht, das Akademie-Institut gemeinsam mit Instituten der Universität in Itzling im Norden Salzburgs anzusiedeln. Es war dies ein Teil der seit Jahren diskutierten Pläne über eine Erweiterung der Universität, die jedoch nie konkretere Formen annahmen. Dieser Vorschlag, ein Institut mit funktionierender Infrastruktur zu schließen und dann an einen anderen Platz in der gleichen Stadt in einen noch zu errichtenden Neubau zu verlegen, war völlig unrealistisch.

Es gab noch weitere Briefe und angeblich auch gegenseitige Besuche, das Fazit ist jedoch, dass es offensichtlich $\mathrm{zu}$ keiner Annäherung der Standpunkte der beiden Herren kam.

\section{Die Entscheidung ist gefallen}

Der erst wenige Tage amtierende Vizepräsident Schuster, der damals auch Vorsitzender des Kuratoriums war, kam am 4. Oktober 2000 nach Salzburg und informierte die Mitarbeiter über den Beschluss des Präsidiums, dass das Institut Ende 2003 geschlossen werden wird. Die Enttäuschung über diese Entscheidung war sehr groß, es herrschte lähmendes Entsetzen, und es gab dementsprechend auch kaum eine Diskussion, die ohnehin sinnlos gewesen wäre. In der Klassensitzung vom 12. Oktober hat dann Vizepräsident Schuster über diesen Besuch berichtet. Als Begründungen für die Schließung wurden u. a. angeführt:

„Auf Grund der Entwicklung in den Life Sciences sei die Standortfrage für ein wissenschaftliches Institut auf diesem Gebiet schon seit längerer Zeit diskutiert worden."

„Um die Voraussetzungen für das wissenschaftliche Arbeiten in Salzburg zu verbessern, wäre ein Ausbau des dortigen Instituts notwendig gewesen." 
„Die Anfrage bezüglich finanzieller Unterstützung an das Land Salzburg sei bis zum heutigen Tag ohne konkrete Zusage geblieben." „Die Akademie plant zudem die Gründung eines neuen Instituts in der Dr.-Bohr-Gasse in Wien. Alle Mitarbeiter aus Salzburg haben die Möglichkeit, sich in Wien zu bewerben.“

Die endgültige Entscheidung, das Institut zu schließen, ist anscheinend in den Sommermonaten dieses Jahres gefallen, wobei offensichtlich weder die Klasse noch die Gesamtakademie in die Diskussionen mit einbezogen wurden. Auch das Kuratorium des Instituts für Molekularbiologie wurde erst im Nachhinein in einer Sitzung am 12. Oktober informiert und vor vollendete Tatsachen gestellt. In den darauf folgenden Jahren wurde von einzelnen Mitgliedern des Kuratoriums mehrfach betont, sie seien nicht konsultiert worden. In späteren Gesprächen kam auch zum Ausdruck, dass diese die Vorgangsweise des Präsidiums nicht billigten, ja z. T. sogar dezidiert ablehnten. Es kam jedoch zu keiner gemeinsamen Initiative gegen den Beschluss des Präsidiums, wobei allerdings fraglich ist, ob eine solche in der damaligen Situation überhaupt eine Chance auf Erfolg gehabt hätte.

Es ist wohl kein Zufall, dass fast zeitgleich, am 29. September 2000, ein Jahr nach der Unterzeichnung des Kooperationsvertrags zwischen der Firma Boehringer-Ingelheim und der Akademie, eine Pressekonferenz stattfand, in der der Entwurf für die neu gegründete IMBA GmbH präsentiert wurde.

Um diese Zeit gab es auch erste Pläne, ein neues Institut für Zellund Entwicklungsbiologie (IZEB) zu gründen, dessen Leitung w. M. Schweizer nach einigem Zögern übernahm. Es war zunächst jedoch nicht klar, welche Personen im IZEB arbeiten und welche Fachrichtungen dort vertreten sein sollten. Ende Jänner 2001 gab es eine Sitzung eines neu gegründeten Ad-hoc-Komitees, dem einzelne Mitglieder des Kuratoriums sowie mehrere Wissenschaftler aus Deutschland und der Schweiz angehörten. Es wurden verschiedene Konzepte für dieses IZEB diskutiert, wobei die Mehrheit die Meinung vertrat, dass in dem in Wien zu errichtenden Institut die „Molekulare Pflanzenbiologie" im Vordergund stehen solle. Dies wurde von Herrn Schweizer auch in einem Konzeptpapier weiter präzisiert, das im Dezember 2001 vom Präsidium und der Gesamtakademie akzeptiert wurde. An Stelle von IZEB wurde der Name GMI (Gregor-MendelInstitut für Molekulare Pflanzenforschung) beschlossen. Dieses Institut wird ab Dezember 2005 in der Dr.-Bohr-Gasse untergebracht sein. Nach längeren Verhandlungen gelang es, das Ehepaar Matzke und die Mitarbeiter dieser Arbeitsgruppe für das GMI zu gewinnen. 


\section{Das langsame Ende}

Die verbleibenden drei Jahre waren sehr mühsam und unerfreulich. Motivation und Produktivität der Mitarbeiter sanken graduell, die Stimmung im Institut verschlechterte sich immer mehr, junge Mitarbeiter verließen das Institut möglichst schnell, neue konnten nicht mehr aufgenommen werden usw. Zudem gab es immer wieder unterschiedliche Nachrichten bzw. Gerüchte über die weitere Vorgangsweise, die aus dem Präsidium, dem Personalreferat und dem Betriebsrat ins Institut diffundierten und die die allgemeine Verunsicherung noch erhöhten. Einmal hieß es, es komme zur Schließung mit Kündigung aller Mitarbeiter; in einem neu gegründeten Institut in Wien sollte dann nur die Gruppe Matzke wieder angestellt werden. Aber auch ein Institut für Molekularbiologie der Pflanzen in Salzburg wurde anscheinend in Betracht gezogen. Eine echte Alternative zur Schließung des Instituts wurde jedoch mit den Vertretern des Instituts nie erörtert. Theoretisch wäre eine Weiterführung mit neuer Zielsetzung eine attraktive Option gewesen, aber das Präsidium hatte einfach andere Pläne.

Letztlich wurden die nach den Pensionierungen von zwei Abteilungsleitern verbleibenden drei Arbeitsgruppen Matzke, Small und Barlow nach Wien transferiert, zwei Juniorgruppen (H. C. Bauer und R. Vlasak) übersiedelten an die Universität Salzburg und werden von der ÖAW fünf weitere Jahre finanziert.

Im Präsidium gab es offensichtlich kein „Ausstiegs-Szenarium“, auch wurde keine Kommission oder dgl. mit der logistisch nicht einfachen Planung und Ausführung der Schließung betraut. Die Mitarbeiter des Instituts hatten vielmehr den Eindruck, dass die anstehenden Probleme im Präsidium punktuell entschieden wurden. Zumindest von außen war jedenfalls keine klare Linie erkennbar, auch nicht bei mehreren Gesprächen mit Frau Hofrat Gatterbauer und Aktuar Schadler. Die Räumung des Instituts war ebenfalls nicht koordiniert worden, wurde aber schließlich von den Mitarbeitern mit großem Einsatz abgewickelt. Am 31. März 2003, exakt 37 Jahre nach der Gründung des Instituts für Molekularbiologie, gab es noch ein großes Abschiedsfest, zu dem auch zahlreiche frühere Mitarbeiter kamen. Und in den folgenden Monaten fiel dann langsam der Vorhang.

Die weitere Nutzung des Hauses in Salzburg wurde zunächst nicht entschieden. Ein Angebot der Medizinischen Privatuniversität Salzburg im Sommer 2003, das Institut zu mieten, wurde trotz der Initiative des neu gewählten, aber noch nicht im Amt befindlichen Vizepräsidenten Matis nicht weiter verfolgt. Das Haus stand dann 
mehr als ein Jahr leer, erst im März 2005 konnte in dieser tristen Situation doch eine einigermaßen befriedigende Lösung gefunden werden. Das Institut wurde an die Universität Salzburg verkauft, die Unterzeichnung des Kaufvertrags fand dann am 2. Mai 2005 statt. In Gegenwart von Landeshauptfrau Burgstaller sollten Präsident Mang und Rektor Schmidinger den Vertrag unterzeichnen. Infolge einer defekten Lokomotive der ÖBB kam Präsident Mang mit großer Verspätung nach Salzburg, und so wurde ich gebeten, i. V. zu unterschreiben. Durch einen seltsamen Zufall war ich demnach auch noch Akteur im Finale der Geschichte des Instituts für Molekularbiologie der Österreichischen Akademie der Wissenschaften. Nach einigen Umbau- und Reparaturarbeiten wurde das Gebäude am 8. November im Rahmen einer Feier wieder eröffnet. Es beherbergt nun den „Fachbereich Molekularbiologie“ des Forschungszentrums „Lebenswissenschaften und Gesundheit" der Universität, ein kleiner Teil wurde an die Medizinische Paracelsus-Universität in Salzburg und die von den früheren Mitarbeitern gegründete Firma ABT vermietet.

Zuletzt ein Zitat, das zeitweise auf der Homepage der ÖAW zu lesen war: „Wir schließen unser bestes Institut, damit Neues, Besseres entsteht". Zweifellos ein wunderlicher Satz, der etliche Fragen aufwirft, vor allem, ob diese Aussage bei einer offenen Diskussion in der Gesamtsitzung von der Mehrheit der Mitglieder der ÖAW unterstützt worden wäre. Aber diese wurden, wie schon erwähnt, vor vollendete Tatsachen gestellt.

Anschrift des Verfassers: Prof. Dr. Günther Kreil, Am Gois 17, 5081 Anif/ Salzburg, Österreich. E-Mail: guenther.kreil@ oeaw.ac.at. 